\title{
Computer Simulation of Multi-dimensional Bone Deformity Correction and Lengthening by Unilateral External Fixator
}

\author{
Yoon Hyuk Kim \\ School of Advanced Technology, Kyung Hee University, 1 Sochon-ni, \\ Kihung-eop, Yongin-shi, Kyongki-do, 449-701, Korea \\ yoonhkim@khu.ac.kr
}

\begin{abstract}
An Analytic and graphic model of a unilateral external fixator and bone system is presented to simulate and visualize the pre-operative planning of three-dimensional adjustments of the fixator joints in osteotomy. An analytic model was developed as a rigid body linkage system, and kinematic chain loop equations were utilized in the adjustment analysis to obtain the necessary rotations and translations at each joint of the fixator to correct bone deformity at the fracture site. Corrections of various deformities, combining various rotations and translations, were simulated to validate the models developed. The presented models achieve the goal of using computer-aided pre-operative planning and intra-operative execution of an osteotomy to correct malalignment and joint dysfunction.
\end{abstract}

\section{Introduction}

Knee osteotomy has been widely recognized as a treatment method for unicompartmental osteoarthritis of the knee [1-5]. This method has been used more frequently in relatively young and active patients with early degenerative changes in the joint [3]. The underlying principles for osteotomy are sound, but one of the causes of undesirable outcome of the treatment relates to a lack of knowledge based pre-operative planning and inadequate execution of the procedure as planned [6-8].

In the pre-operative planning stage for osteotomy, one crucial factor in obtaining good clinical results is the accurate determination of femoral-tibial alignment in order to redistribute joint contact pressure [6,9-11]. Achieving the desired corrective angle is also difficult and unpredictable in preventing deformity recurrence. To accomplish accurate osteotomy, an examination of three-dimensional deformity, initial osteotomy plane orientation, and subsequent angular and translational adjustment, taking into account bone segment fixation stability must be carefully planned and executed.

External fixation has been widely used in bone segment stabilization following osteotomy [12-14]. External fixation has a distinct advantage in its ability to directly adjust the bone deformity intra-operatively in one setting or post-operatively adjust bone segments through gradual distraction at the osteotomy site. Furthermore, it al- 
lows continuous monitoring of bone and joint alignment to avoid residual deformity in rotation and translation. To the author's knowledge, however, there has been no study conducted to evaluate the existing fixator's ability to achieve deformity correction based on certain application configurations and also determine the precise fixator joint adjustments in order to execute the correction plan accurately.

The objective of this study was to simulate a three-dimensional knee osteotomy of different bone and joint deformities using a unilateral external fixator to accomplish the required alignment correction. In order to determine the necessary fixator joint adjustments, the bone segments, the osteotomy site and the external fixator were modeled as a multi-link kinematic mechanism. The associated kinematic analysis under given bone deformities and fixator application configurations provided the degree of translation and rotation to be performed in each fixator joint in order to precisely correct a bone deformity. A computer graphic model of both the fixator and tibia were developed to visualize and validate the analysis results and to evaluate the osteotomy execution plan under external immobilization and adjustment. The presented technology assures the precise execution of the desired deformity correction.

\section{Materials and Methods}

\subsection{Mathematical and Computer Graphic Model}

A unilateral external fixator for deformity correction (DynaExtor ${ }^{\circledR}$, Bkmeditech, Korea) was used in this analysis. The fixator model is composed of eight pins inserted into the bones, a central telescoping rod, two pin clamps, two rotational rod clamps, and two connecting bodies which have two revolute rotations, respectively (Fig. 1). The analytical representation of the motion of each link of the fixator and the bone segments can be expressed as a kinematic linkage system interconnected by five revolute and one prismatic joints, thus having 6 degrees-of-freedom (DOF). The central rod of the fixator has a range of $5 \mathrm{~cm}$ of compression/distraction, and the rotational clamps have unlimited rotation along the long axis of its body. Both the distal connecting body and the proximal connecting body reach their full range of varus/valgus angulation at $45^{\circ}$ of flexion/extension. In the model, all joints of the fixator begin in their neutral positions, with the exception of the lower revolute joint, which is rotated $-10^{\circ}$ in y-direction. Other geometric dimensions were measured and defined to facilitate the kinematic analysis (Fig. 1).

The bone was modeled as a cylinder, and the osteotomy cutting-plane was defined at diaphyseal area of the bone. The simple frontal plane angular correction was accomplished by rotating the distal bone segment with respect to the AP axis through the medial edge of the proximal bone segment in the frontal plane based on the specified wedge angle. The proximal and distal bone segments were modeled as two rigid bodies, with the distal bone segment fixed to the origin of the global coordinate system. Other three-dimensional osteotomy corrections including translation were defined as the input data specified by three rigid body Eulerian angle rotations and three translations, which were to be achieved by adjusting the fixator joints. The computer graphic 
model of the bone-fixator system was developed using the software SolidWorks ${ }^{\text {TM }}$ (Solidworks, Concord, MA, USA) in order to visualize the computed results of joint rotation and translation required to achieve the desired osteotomy correction.

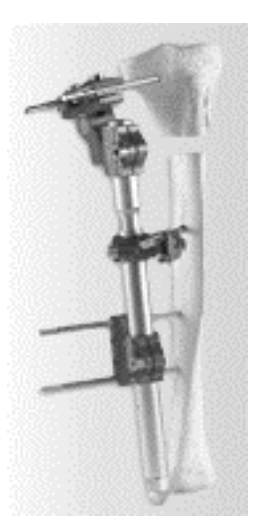

(a)

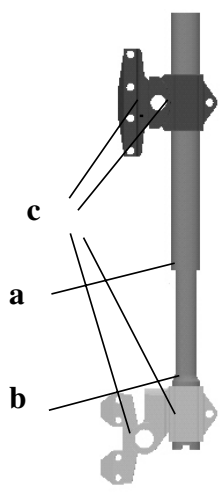

(b)

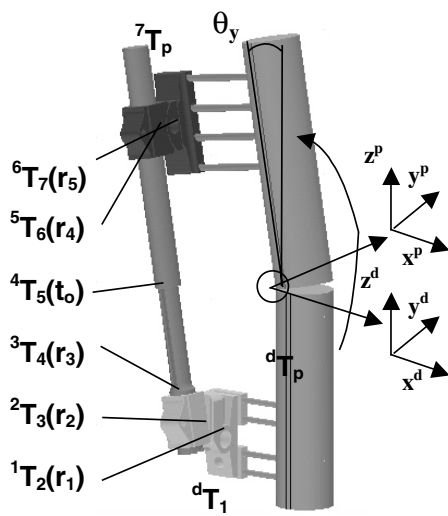

(c)

Fig. 1. The real, (a), and the graphic, (b), models of the DynaExtor ${ }^{\otimes}$ external fixator (Bkmeditech, Korea ): "a" - the central telescoping rod, "b" - the rotational joints, "cc" - the revolute joints. The geometric parameters used in this paper were referred from the company catalog. (c) The tibia-fixator system for 3-D deformity correction with the transformation matrices, ${ }^{\mathrm{i}} \mathbf{T}_{\mathrm{i}+1}$, and all the joint variables indicated on the DynaExtor fixator. The original varus deformity, $\theta_{\mathrm{y}}$, was defined as $-10^{\circ}$ of y-rotation

\subsection{Inverse Kinematics Analysis}

In order to define deformity parameters between the two bone segments, a 4 x 4 homogeneous transformation matrix was utilized to express the kinematic loop equations of the fixator-tibia system [15]. The rotational sequences at the fracture site follows the $x-y$ '-z" Euler-angle system [15]. The global coordinate system was fixed to the distal segment of the tibia (Fig. 1). All local joint coordinate systems were defined identically to the global coordinate system. In the mathematical analysis, ${ }^{d} \mathbf{T}_{\mathrm{p}}$ is the transformation matrix from the proximal tibial segment to the distal segment, was expressed by the matrix or loop equation shown below:

$$
{ }^{\mathrm{d}} \mathbf{T}_{\mathrm{p}}={ }^{\mathrm{d}} \mathbf{T}_{1} \cdot{ }^{1} \mathbf{T}_{2} \cdot{ }^{2} \mathbf{T}_{3} \cdot{ }^{3} \mathbf{T}_{4} \cdot{ }^{4} \mathbf{T}_{5} \cdot{ }^{5} \mathbf{T}_{6} \cdot{ }^{6} \mathbf{T}_{7} \cdot{ }^{7} \mathbf{T}_{\mathrm{p}} \cdot
$$

${ }^{\mathrm{d}} \mathbf{T}_{1}$ and ${ }^{7} \mathbf{T}_{\mathrm{p}}$ represent the rigid body translations of the local coordinate systems between the bone segments and the pin clamps (Fig. 2). The matrices ${ }^{1} \mathbf{T}_{2},{ }^{2} \mathbf{T}_{3},{ }^{5} \mathbf{T}_{6}$ and ${ }^{6} \mathbf{T}_{7}$ are pure rotations at the revolute joints. ${ }^{3} \mathbf{T}_{4}$ represents the axial rotation at the lower rotational clamp, and ${ }^{4} \mathbf{T}_{5}$ represents translation between the prismatic joints at 
the central telescoping rod. After substituting the unknown fracture ( ${ }^{\mathrm{d}} \mathbf{T}_{\mathrm{p}}$ ) and fixator geometric parameters into the transformation matrices, the six unknown joint variables, $\mathbf{r}_{1}, \mathbf{r}_{2}, \mathbf{r}_{3}, \mathbf{r}_{4}, \mathbf{r}_{5}$ and $\mathbf{t}_{\mathbf{o}}$, can be determined by solving the matrix Eq. (1), where, $\mathbf{r}_{1}$, $\mathbf{r}_{2}, \mathbf{r}_{3}, \mathbf{r}_{4}$ and $\mathbf{r}_{5}$ are the revolute variables, and $\mathbf{t}_{\mathbf{o}}$ is the translational variables, respectively [15] (Fig. 1). The resulting systems of non-linear equations were solved using the nonlinear least square optimization method (MATLAB ${ }^{\mathrm{TM}}$, Mathworks, MA, USA) [15]. In the optimization process, the limit of range in each joint was set as a constraint equation.

\subsection{Simulation and Graphic Validation}

To validate the presented analysis method, a correction of $10^{\circ}$ varus deformity was simulated using graphic animation. In addition, anterior (-y-axis) translation, medial (-X-axis) translation, bone lengthening (z-translation), external rotation (z-rotation), and flexion (x-rotation) of the proximal segment in reference to the distal segment were combined with the varus deformity correction in order to fully illustrate the 3-D fixator adjustability and the analysis method used to achieve accurate alignment correction during treatment. Based on analysis results and graphic animation, the deformity correction planning process can be clearly visualized and evaluated.

\section{Results}

In order to demonstrate the adjustability of the fixator and the wide range of osteotomy options in 3-D space, both simple and complex malalignment cases were considered. Computer graphic models of the fixator and the bone segments were used to illustrate their pre-adjustment and corrected configurations.

In the case of a $10^{\circ}$ varus deformity, the solution of the fixator joint variables for a closed wedge osteotomy site was obtained for the DynaExtor ${ }^{\circledR}$ fixator, as $\left(\mathbf{r}_{1}=0^{\circ}, \mathbf{r}_{2}=\right.$ $5.8^{\circ}, \mathbf{r}_{3}=0^{\circ}, \mathbf{r}_{4}=0^{\circ}, \mathbf{r}_{5}=4.2^{\circ}, \mathbf{t}_{\mathbf{o}}=10.8 \mathrm{~mm}$ ) (Table 1). By rotating the revolute joints associated with $\mathbf{r}_{2}$ and $\mathbf{r}_{5}$ along the $y$-axis and translating $\mathbf{t}_{\mathbf{0}}$, the fixator closes the wedge gap perfectly. The upper and lower bounds imposed on the rotational and prismatic fixator joints based on the fixator design constraints improve the solution convergence. According to the analysis results, the graphic simulation showed a perfect reduction of the wedge gap achieved by the above changes in the fixator joint variables (Fig. 2b).

In the case of a $10^{\circ}$ varus deformity with $5 \mathrm{~mm}$ medial translation of the proximal segment in reference to distal osteotomy surface, the resulting solutions of the fixator joint variables for the osteotomy site were: $\mathbf{r}_{1}=0^{\circ}, \mathbf{r}_{2}=4.2^{\circ}, \mathbf{r}_{3}=0^{\circ}, \mathbf{r}_{4}=0^{\circ}, \mathbf{r}_{5}=5.8^{\circ}, \mathbf{t}_{\mathrm{o}}$ $=11.5 \mathrm{~mm}$ (Table 1$)$. When knee joint laxity needs to be increased, additional medial translation can be incorporated into the osteotomy in order to balance the knee ligaments. In this case, the same two revolute joints, which were involved in pure osteotomy reduction, now functioned to perform combined rotation and translation (Fig. 2c). 


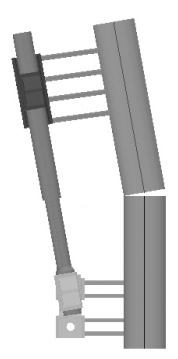

(a)

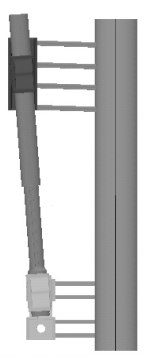

(b)

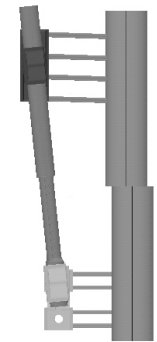

(c)

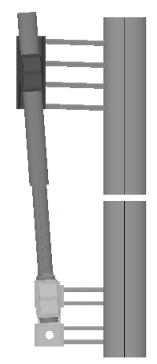

(d)

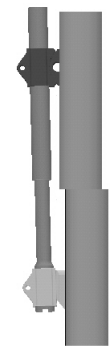

(e)

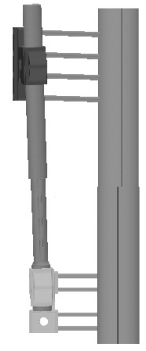

(f)

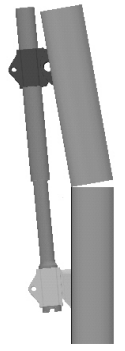

(g)

Fig. 2. (a) The bone-fixator configuration before the correction of deformity. (b)-(g) shows the bone-fixator configuration after the correction for pure correction; (b): correction with $5 \mathrm{~mm}$ medial translation, (c): correction with bone lengthening, (d): correction with $5 \mathrm{~mm}$ anterior translation, (e): correction with $10^{\circ}$ external rotation, (f): and correction with $10^{\circ}$ flexion, respectively. The results showed that different joint combinations contributed to perform the correction processes for different deformities and planning using the presented techniques would be useful before the intra-operation to obtain the accurate execution results

In the case of a $10^{\circ}$ varus deformity with $5 \mathrm{~mm}$ bone lengthening, the solutions of the fixator joint variables at the osteotomy site were: $\mathbf{r}_{1}=0^{\circ}, \mathbf{r}_{2}=5.9^{\circ}, \mathbf{r}_{3}=0^{\circ}, \mathbf{r}_{4}=0^{\circ}, \mathbf{r}_{5}$ $=4.1^{\circ}, \mathbf{t}_{\mathbf{o}}=16.7 \mathrm{~mm}$ (Table 1 ). In this case, the result added $5 \mathrm{~mm}$ of the distal prismatic joint, $\mathbf{t}_{\mathbf{0}}$, to the case of pure $10^{\circ}$ of varus deformity correction. The graphic model shows the $5 \mathrm{~mm}$ of gap at the osteotomy site (Fig. 2d).

In the case of a $10^{\circ}$ varus deformity with $5 \mathrm{~mm}$ anterior translation at the osteotomy site, the solutions of the fixator joint variables at the osteotomy site were: $\mathbf{r}_{1}=1.5^{\circ}, \mathbf{r}_{2}$ $=5.8^{\circ}, \mathbf{r}_{3}=0.1^{\circ}, \mathbf{r}_{4}=-1.5^{\circ}, \mathbf{r}_{5}=4.7^{\circ}, \mathbf{t}_{\mathbf{o}}=11.1 \mathrm{~mm}$ (Table 1$)$. In order to obtain the anterior translation of the bone segments, the two revolute joints $\mathbf{r}_{1}$ and $\mathbf{r}_{\mathbf{4}}$ were rotated in the direction of $\mathrm{X}$-axis, with the same magnitudes but opposite signs. Fig. 2e shows the lateral view (x-axis) of the fixator-bone system to validate the anterior translation of the system.

The case of a $10^{\circ}$ varus deformity with $10^{\circ}$ external rotation at the osteotomy site required all fixator joints to move significantly in order to perform the correction: $\mathbf{r}_{1}=$ $3.7^{\circ}, \mathbf{r}_{2}=8^{\circ}, \mathbf{r}_{3}=-9.6^{\circ}, \mathbf{r}_{4}=-3.4^{\circ}, \mathbf{r}_{5}=2.6^{\circ}, \mathbf{t}_{\mathbf{o}}=11.4 \mathrm{~mm}$, (Table 1). This result clearly 
illustrates the capability of the fixator and the ability of the analysis software to generate 3-D movement at Knee osteotomy site. The Fig. $2 \mathrm{f}$ shows perfect adjustment of the bone segments as well as the axial rotations between the proximal and the distal osteotomy planes.

In the combined rotational deformity case with $10^{\circ}$ varus and $10^{\circ}$ flexion, the solution of the fixator joint variables osteotomy site were determined to be $\mathbf{r}_{1}=5.1^{\circ}, \mathbf{r}_{2}$ $=5.8^{\circ}, \mathbf{r}_{3}=-0.4^{\circ}, \mathbf{r}_{4}=4.9^{\circ}, \mathbf{r}_{5}=4.1^{\circ}, \mathbf{t}_{\mathbf{o}}=10.8 \mathrm{~mm}$ (Table 1). To obtain the flexion effect between the bone segments, the two rotational joints in the medial-lateral direction, $\mathbf{r}_{1}$ and $\mathbf{r}_{4}$, together rotated about $10^{\circ}$. In the graphic model, the rotations are clearly demonstrated between the two segments in the lateral view (Fig. 2g). With the use of an appropriate planning program that can define the correction required in all planes, the presented analysis technique can determine the precise adjustment required of each joint to achieve the specified osteotomy.

Table 1. Solutions of the DynaExtor ${ }^{\circledast}$ external fixator joint under $10^{\circ}$ varus deformity correction combined various translations and rotations based on closed wedge osteotomy

\begin{tabular}{|c|c|c|c|c|c|c|}
\hline \multirow{2}{*}{$\begin{array}{l}\text { Deformity Pa- } \\
\text { rameters }\end{array}$} & \multicolumn{6}{|c|}{ Fixator Joint Variables } \\
\hline & $\left(^{\circ}\right)$ & $\left(^{\circ}\right)^{\mathbf{r}_{2}}$ & $\left(^{\circ} \mathbf{r}_{3}\right.$ & $\left({ }^{\circ}\right)$ & $\begin{array}{c}\mathbf{r}_{5} \\
\left({ }^{\circ}\right)\end{array}$ & $\begin{array}{r}\mathbf{t}_{\mathbf{o}} \\
(\mathrm{mm})\end{array}$ \\
\hline $10^{\circ}$ varus deformity & 0.0 & 5.8 & 0.0 & 0.0 & 4.2 & 10.8 \\
\hline $\begin{array}{l}10^{\circ} \text { varus }+5 \mathrm{~mm} \\
\text { medial translation }\end{array}$ & 0.0 & 4.2 & 0.0 & 0.0 & 5.8 & 11.5 \\
\hline $\begin{array}{l}10^{\circ} \text { varus }+5 \mathrm{~mm} \\
\text { bone lengthening }\end{array}$ & 0.0 & 5.9 & 0.0 & 0.0 & 4.1 & 16.7 \\
\hline $\begin{array}{l}10^{\circ} \text { varus }+5 \mathrm{~mm} \\
\text { anterior translation }\end{array}$ & 1.5 & 5.8 & 0.1 & -1.5 & 4.7 & 11.1 \\
\hline $\begin{array}{l}10^{\circ} \text { varus }+10^{\circ} \\
\text { external rotation }\end{array}$ & 3.7 & 8.0 & -9.6 & -3.4 & 2.6 & 11.4 \\
\hline $\begin{array}{l}10^{\circ} \text { varus }+10^{\circ} \\
\quad \text { flexion }\end{array}$ & 5.1 & 5.8 & -0.4 & 4.9 & 4.1 & 10.8 \\
\hline
\end{tabular}

\section{Discussion}

Unicompartmental osteoarthritis (OA) of the knee is a common disorder due to the weight bearing, high-mobility and stability required of the joint [1-4]. Altered limb alignment in a knee with unicompartmental arthritis causes the load to shift towards the affected compartment, thus causing further joint deformity and degenerative changes. Therefore, joint alignment and loading redistribution are considered to be the key biomechanical justifications for knee osteotomy. 
Despite the many advantages of knee osteotomy, it has not been favored as the treatment of choice even when clinical indications are favorable for such treatment because of several possible complications. Improper pre-operative planning and poor execution of the surgical plan have been identified as causes of deformity and pain recurrence [7]. In order to obtain good results, computer-assisted 3-D preoperative planning and execution of knee osteotomy is necessary [6, 9-12]. If an external fixator is used as the adjustment and stabilization device in a knee osteotomy, gradual and precise correction of the residual deformity at the osteotomy site can be accomplished by rotating and translating the fixator joints gradually on an adjustment plan.

When flexional/extensional deformities are combined with internal/external rotational malalignments, additional factors must be considered during preoperative planning, execution, and postoperative residual deformity readjustment. During osteotomy, there is a possibility of the tilting of the osteotomy line in the anterior/posterior direction due to the difficulty of saw blade angle control [8]. To maintain sagittal plane orientation of the tibial plateau it is important to avoid residual flexion/extension deformity of the tibia, since joint articular surface motion is improved when the normal posterior tilt of the tibial plateau is recovered. Furthermore, an intended internal/external rotation of the foot can be generated when the axis of the osteotomy closing is not perpendicular to the frontal plane. In addition to the intra-operative technical difficulties of knee osteotomy, rotational deformities from cerebral palsy or a congenital disease in which 3-D osteotomy is needed have been reported [16]. The current analysis software and graphic models can facilitate correction of all deformities at any level of the long bone by providing accurate and reliable corrections through fixator joints or robotic arms both in one-step correction and internal fixation or in gradual correction through callus distraction under external fixation.

The results of current analysis and simulation show that knee osteotomy can be achieved by adjusting a combination of translations and rotations of the fixator joints. Furthermore, the current analysis and simulation techniques can be used to determine the adjustment capability of the current design of any fixator, evaluate its performance during surgical treatment, and review its clinical application in individual patients. These kinds of tools can also provide the guidelines for a desirable biomechanical environment at fracture sites that enhances fracture union and bone maturation after lengthening. By integrating its use with image-guided and robotics-assisted tools, a fully controlled, 3-D, computer-aided osteotomy surgery and rehabilitation procedure can be established.

\section{Summary}

This paper presents a kinematic analysis and a graphic simulation tool for osteotomy under external fixation for complex 3-D bone deformities. Using the developed analysis tool, the required amount of the rotations and translations of the fixator joints were determined to reduce the given deformities in osteotomy with combined 3-D rotations and translations at the osteotomy site. In addition, the graphic model of the bonefixator system developed in this study demonstrates its capabilities in validating analy- 
sis results and simulating the pre-operative planning of osteotomy. Furthermore, these tools can be used to evaluate the existing planning methods of osteotomy, enhancing their performance and facilitating changes in the design configuration for the external fixator.

Acknowledgement. This study was supported in part by Res. Inst. of Med. Instr. \& Reh. Eng., Kosef, Korea.

\section{References}

1. Coventry, M.B.: Upper tibial osteotomy for osteoarthritis. J. Bone Joint Surg. 67-A (1987) 1136-1140

2. Coventry, M.B.: Proximal tibial varus osteotomy for osteoarthritis of the lateral compartment of the Knee. J. Bone Joint Surg. 69-A (1987) 32-38

3. Fu, F.H., Harner, C.D., Vince, K.G.: The Knee Surgery Vol 2. Williams \& Wilkins (1994)

4. Grelsamer, R.P.: Current concepts review: Unicompartmental osteoarthrosis of the Knee. J. Bone Joint Surg. 77-A (1995) 278-287

5. Marti, R.K., Verhagen, R.A.W., Kerkhoffs, G, Moojrn, T.M.: Proximal tibial varus osteotomy. J. Bone Joint Surg. 83-A (2001) 164-170

6. Hsu, R.W.W., Himeno, S., Coventry, M.B., Chao, E.Y.S.: Normal axial alignment of the lower extremities and load-bearing distribution at the Knee. Clin. Orthop. Related Res. 255 (1990) 215-227

7. Jackson, M., Sarangi, P.P., Newman, J.H.: Revision total Knee arthroplasty. J. Arthroplasty 9 (1994) 539-542

8. Ellis, R.E., Tso, C.Y., Rudan, J.F., Harrison, M.M.: A surgical planning and guidance system for high tibial osteotomy. Comput. Aided Surg. 4 (1999) 264-274

9. Paley, D., Tetsworth, K.: Mechanical axis deviation of the lower limbs; Preoperative planning of uniapical deformities of the tibia and femur. Clin. Orthop. Related Res. 280 (1992) $48-64$

10. Chao, E.Y.S., Sim, F.H.: Computer-aided preoperative planning in Knee osteotomy. The Iowa Orthop. J. 15 (1995) 4-18

11. Chao, E.Y.S., Neluheni, E.V.D., Hsu, R.W.W., Paley, D.: Biomechanics of malalignment. Orthop. Clin. North America 25 (1994) 379-386

12. Paley, D., Chaudray, M., Pirone, A.M., Lentz, P., Kautz, D.: Treatment of malunions and mal-nonunions of the femur and tibia by detailed preoperative planning and the Ilizarov techniques. Orthop. Clin. North America 21 (1990) 667-91

13. Choi, I.H., Chung, C.Y., Cho ,T.J., Park, S.S.: Correction of genu recurvatum by the Ilizarov method. J. Bone Joint Surg. 81-B (1999) 769-774

14. Larsen, S.T., Magyar, G., Onsten, I., Ryd, L., Lindstrand, A.: Upper tibial valgus osteotomy using a dynamic external fixator. J. Bone Joint Surg. 80-B (1998) 295-297

15. Kim, Y.H., Inoue, N., Chao, E.Y.S.: Kinematic simulation of fracture reduction and bone deformity correction under unilateral external fixation. J. Biomech. (2002) 1047-1058

17. Healy, W.L., Anglen, J.O., Wasilewski, S.A., Krackow, K.A. Distal femoral varus osteotomy. J. Bone Joint Surg. 70-A (1988) 102-109 